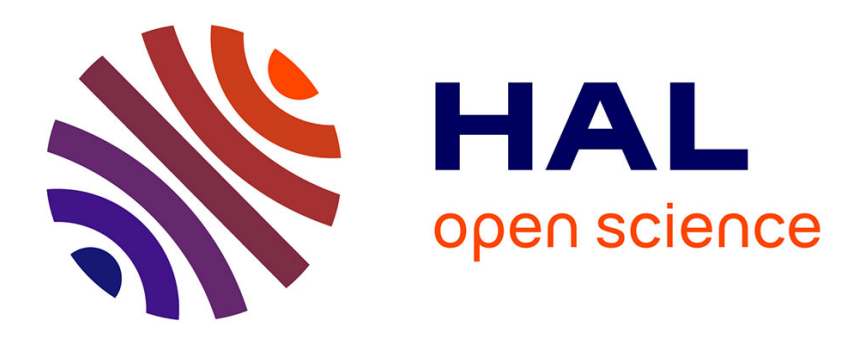

\title{
Ancrer le capital dans les flux logistiques : la financiarisation de l'immobilier logistique
}

Nicolas Raimbault

\section{To cite this version:}

Nicolas Raimbault. Ancrer le capital dans les flux logistiques : la financiarisation de l'immobilier logistique. Revue d'économie régionale et urbaine, 2016, Février (1), pp.131-154. 10.3917/reru.161.0131. hal-02162749

\section{HAL Id: hal-02162749 \\ https://hal.science/hal-02162749}

Submitted on 15 Aug 2019

HAL is a multi-disciplinary open access archive for the deposit and dissemination of scientific research documents, whether they are published or not. The documents may come from teaching and research institutions in France or abroad, or from public or private research centers.
L'archive ouverte pluridisciplinaire HAL, est destinée au dépôt et à la diffusion de documents scientifiques de niveau recherche, publiés ou non, émanant des établissements d'enseignement et de recherche français ou étrangers, des laboratoires publics ou privés. 


\title{
Ancrer le capital dans les flux logistiques : la financiarisation de l'immobilier logistique
}

\author{
Anchoring capital into logistics flows: the \\ financialization of logistics real estate
}

\author{
Nicolas RAIMBAULT \\ Université Paris-Est ; IFSTTAR - AME/SPLOTT \\ nicolas.raimbault@ifsttar.fr \\ Auteur correspondant
}

\begin{abstract}
Mots-clés
Action publique, Financiarisation, Immobilier logistique, Périurbanisation, Privatisation.
\end{abstract}

Keywords

Financialization, Logistics Real Estate, Peri-urbanization, Privatization, Public action.

\section{Résumé}

Si la financiarisation de l'immobilier résidentiel, commercial et de bureau a été particulièrement investie par la littérature académique, un produit immobilier particulier a moins attiré l'attention: l'immobilier logistique. Or, sous l'impulsion de développeursgestionnaires de fonds internationaux spécialisés, ce dernier suit une trajectoire de financiarisation particulière qui offre un regard complémentaire sur les logiques contemporaines de la production urbaine. Dans cet article, nous analysons les ressorts de la financiarisation de l'entrepôt et nous mettons en lumière ses conséquences sur l'action publique locale. Nous montrons ainsi qu'en apportant une capacité à développer des zones logistiques dans des territoires qui en sont dépourvus, elle conduit à privatiser les politiques locales d'aménagement et, par suite, une grande part des outils de régulation de ce développement économique. De cette façon, nous mettons en évidence tout l'intérêt d'analyser la financiarisation de la production urbaine par un regard précis sur les politiques locales.

\footnotetext{
Abstract

The financialization of residential real estate, shopping malls and office properties has been particularly studied by academic literature. However, a specific real estate property hasn't drawn too much attention: logistics real estate. Yet, this latter, due to the emergence of specialized international real estate developers-investors, follows an original financialization path, which reveals new trends of current city-making logics. In this paper, the mechanisms of the financialization of logistics real estate are analyzed and its consequences on local public actions are highlighted. We demonstrate that this financialization, while bringing the capacity to develop logistics zones in territories whose governments don't have the capacity to manage on their own such projects, leads to the privatization of local planning policies and, thus, of a large part of the regulation tools of this economic development. This way, we highlight the
} 
strong interest of analyzing the financialization of city-making from the perspectives of local policies.

\section{$-1-$ \\ Introduction}

L'analyse de la financiarisation de l'immobilier résidentiel, commercial et de bureau, c'està-dire sa transformation en un actif financier, a été particulièrement investie par la littérature académique (Theurillat et al, 2010, 2014 ; NAPPI-ChOUlet, 2013 ; DAVID et HALBERT, 2013 ; HALBERT et al, 2014). Ces travaux mettent en évidence une dynamique puissante de financiarisation de la production urbaine (RUTLAND, 2010 ; LORRAIN, 2011a ; HALBERT 2013). Un produit immobilier particulier a moins attiré l'attention : l'immobilier logistique (à l'exception de HESSE, 2004). Il s'agit du marché des entrepôts, souvent aussi nommés platesformes logistiques ou centres de distribution, qui se sont multipliés en périphérie des métropoles (DABLANC ET FREMONT, 2015). Or, ce produit immobilier suit une trajectoire de financiarisation particulière qui offre un regard complémentaire sur les logiques contemporaines de la production urbaine.

Le marché des entrepôts s'est récemment constitué comme une branche particulière de l'industrie immobilière, dominée par des grandes firmes internationales, spécialisées dans l'immobilier logistique et exerçant à la fois les métiers de développeur et d'investisseur (Hesse, 2004 ; RAIMBAUlt, 2013a). Cette structure s'oppose donc à celle des autres marchés immobiliers au sein desquels les investisseurs ne souhaitent pas s'impliquer dans la production concrète du bâti, laissant cette tâche à des promoteurs distincts, divers et souvent locaux (HALBERT, 2013), jouant le rôle d' «acteurs-ancreurs » (THEURILlat, 2011). Ainsi les firmes de l'immobilier logistique, intégrant investissement et développement des bâtiments, parviendraient-elles à se passer de ces «ancreurs » en mêlant expansion globale et participation à l'action publique locale (RAIMBAULT, 2013b).

Comment expliquer les particularités du marché immobilier logistique ? Pourquoi la financiarisation de cet immobilier se traduit-elle différemment de celle des autres marchés immobiliers? Nous montrons dans cet article que l'un des principaux champs d'explication tient aux modalités de la réception locale des projets immobiliers logistiques. Les entrepôts étant construit dans des espaces plus périphériques que la majorité des autres projets immobiliers, les règles s'avèrent être différentes de celles qui régissent les projets immobiliers des espaces urbains plus denses. Par suite, en mettant l'accent d'une part sur l'importance de l'action publique pour expliquer les modalités de la financiarisation des marchés immobilier et de l'autre sur les conséquences de cette financiarisation sur l'action publique locale, notre travail propose des pistes de réflexions plus théoriques quant à l'étude de la financiarisation de la production urbaine. Nous souhaitons montrer l'intérêt de prolonger l'analyse par les réseaux d'acteurs permettant la commutation du capital vers des territoires particuliers (DAVID et HALBert, 2014 ; HALbert et RouANET, 2014) par un regard précis sur les politiques locales (GUIRONNET et HALBERT, 2014). Dans ce cadre, les théories à portée moyenne des « régimes urbains » (STONE, 1993) et des «coalitions de croissance » (LOGAN et MoLOTCH, 1987) nous semblent à même de montrer non seulement comment les autorités publiques accommodent in fine les critères des investisseurs (GUIRONNET et al, 2015) mais aussi comment les acteurs financiers et immobiliers pèsent concrètement sur l'action publique de ces territoires, pilotent une part des politiques d'aménagement et de développement économique de ces territoires. Autrement dit, cette perspective permet de passer de 
l'explication des modalités de l'atterrissage des capitaux en un lieu à l'analyse des mutations des modes de gouvernance consécutives à la financiarisation de la ville.

Nous exposerons dans un premier temps les logiques de la financiarisation de l'immobilier logistique et le profil des firmes qui l'animent. Nous expliquerons ensuite le succès des développeurs-gestionnaires de fonds internationaux. Enfin, nous analyserons les modalités de la participation de ces firmes aux politiques publiques de développement de zones logistiques autour d'études de cas de la métropole parisienne (RAIMBAULT, 2014).

\section{$-2-$}

\section{La financiarisation de l'immobilier logistique}

Dans cette première partie, nous étudierons les modalités d'émergence du marché spécifique de l'immobilier logistique. Nous mettrons en évidence le poids d'acteurs particuliers : des développeurs-gestionnaires de fonds internationaux.

\subsection{La construction récente d'un marché immobilier logistique}

L'immobilier logistique est une branche récente de l'immobilier d'entreprise. Il répond à d'importants besoins : entre 1985 et 2009 il s'est construit en France autant de surface d'entrepôts que de commerces et de bureaux ${ }^{1}$. Jusqu'au milieu des années 1990, la construction, l'investissement ainsi que la gestion immobilière des entrepôts étaient généralement pris en charge par les utilisateurs d'entrepôts eux-mêmes (HESSE, 2004)². Puis, progressivement, les enjeux immobiliers de l'implantation logistique ont été externalisés à un marché de promotion. Entre 1994 et 2007, 61\% des surfaces d'entrepôts auraient été construites par des promoteurs immobiliers ${ }^{3}$ et non par les utilisateurs des entrepôts ${ }^{4}$. Cette proportion est aujourd'hui plus grande encore. En devenant ainsi un produit immobilier, progressivement standardisé (l'entrepôt de classe A), l'entrepôt est aussi devenu un produit d'investissement représentant en moyenne $10 \%$ du total investi en immobilier dans les années 2000. Cette évolution est récente et massive. Alors qu'avant 1999 les investisseurs ne représentaient que $30 \%$ des propriétaires des entrepôts construits, ce chiffre passe à $67 \%$ entre 1999 et 2001 et même à $75 \%$ depuis $2001^{5}$. Le recours aux investisseurs, c'est-à-dire la préférence des utilisateurs d'entrepôts pour la location, concerne à la fois les bâtiments nouvellement construits et leur patrimoine existant ${ }^{6}$.

\footnotetext{
${ }^{1}$ C'est-à-dire plus de 96000000 de m². Source : sit@del2, SoeS, 2012.

${ }^{2}$ On distingue deux types d'utilisateurs: les chargeurs et les logisticiens. Les chargeurs sont les propriétaires des marchandises. Il s'agit principalement d'industriels ou d'entreprises de la grande distribution. Ils peuvent décider d'externaliser leurs activités logistiques (transport, entreposage, pilotage de la chaîne d'approvisionnement) à des prestataires spécialisés : les logisticiens.

${ }^{3}$ Le rôle du promoteur est d' « assurer la gestion d'un capital monétaire dans sa phase de transformation en bien logement ou tout autre produit fini. Il se trouve investi du suivi du programme et de la coordination des opérations des différents acteurs » (GRANELLE, 1998, p. 114).

${ }^{4}$ Source : Observatoire de l'immobilier logistique et du supply chain management (OBLOG), 2007, État du Parc Immobilier Logistique en France, Paris, Décembre.

${ }^{5}$ Source : BRETAGNOLLe, 2012 à partir de Gse.

${ }^{6}$ Les chargeurs et les logisticiens sont de plus en plus nombreux à revendre les entrepôts qu'ils possèdent à des investisseurs immobiliers pour en devenir de simples locataires (lease back).
} 
Parmi les mécanismes expliquant la naissance de ce nouveau marché immobilier, c'est-àdire la préférence des utilisateurs d'entrepôts pour la location plutôt que la propriété, la recherche de plus grande flexibilité spatiale de la part de ces entreprises est certainement centrale (RAIMBAULT et al, 2013). Dans leur recherche permanente d'optimisation et de rationalisation, les chargeurs et les prestataires renouvellent de manière régulière le nombre et la localisation de leurs entrepôts. L'implantation logistique est un élément important de la performance logistique (MASSON et PETIOT, 2014). Cette préférence est encore renforcée par les effets de la sous-traitance des activités logistiques. En effet, les chargeurs externalisant leurs activités logistiques ne proposent généralement aucune solution immobilière aux prestataires logistiques. Il revient à ce dernier de trouver un bâtiment dans lequel il puisse s'acquitter de la prestation logistique demandée. Etant donné les durées des contrats logistiques, généralement d'une ou quelques années, tout concourt à ce que les utilisateurs d'entrepôt recherchent les baux les plus courts possibles, et donc à ce qu'ils se reposent sur des investisseurs. Par ailleurs, le marché de l'immobilier logistique a aussi profité de la règlementation des Installations Classées pour la Protection de l'Environnement (ICPE). En effet, une partie du parc d'entrepôts construit dans les années 1970 et 1980 est ainsi devenu obsolète. Il était nécessaire de construire un nouveau parc, fournissant de ce fait un objet à ce marché en émergence ainsi que l'opportunité d'imposer le standard de l'entrepôt de classe A.

L'immobilier logistique constitue ainsi un marché récent. Il est encore en cours d'institutionnalisation et de stabilisation. Cet actif n'a pas encore connu un cycle immobilier complet contrairement aux autres actifs immobiliers. Les caractéristiques fondamentales de ce marché immobilier logistique sont les suivantes. La demande rassemble un petit nombre d'entreprises: grands chargeurs (notamment grande distribution et e-commerce) et prestataires logistiques généralement de taille nationale, européenne ou mondiale. L'immobilier logistique est un actif à fort rendement locatif (le meilleur) mais à faible rendement capitalistique ${ }^{7}$. Ces singularités expliquent largement la dynamique de structuration du marché autour d'un rôle croissant des firmes intégrées et globalisées et sa financiarisation avec des fonds d'investissement. Ce sont tout particulièrement les firmes porteuses de cette dynamique qui sont en train de définir les règles de ce marché en cours de stabilisation.

\subsection{Les firmes de l'immobilier logistique : le poids des investisseurs}

A partir du milieu des années 1990, l'entrepôt est devenu un produit proposé par de véritables promoteurs. Surtout, un marché de l'investissement immobilier logistique a émergé et a restructuré en retour le marché de la promotion, conduisant à l'apparition de développeurs-gestionnaires de fonds.

L'entrepôt a suscité l'intérêt des principaux promoteurs français, tels que Nexity ou Bouygues. Il s'agit de promoteurs généralistes qui revendent les immeubles construits à des investisseurs tiers ou aux utilisateurs. Cependant, les principaux « développeurs ${ }^{8}$ logistiques ne se définissent pas comme promoteurs. Ils développent presqu'exclusivement pour leur investissement propre. Leur modèle économique relève davantage de l'investissement que de la promotion. De plus, à partir de 2008, le marché de l'immobilier logistique s'est restructuré presqu'exclusivement autour de ces quelques investisseurs et développeurs-investisseurs : «la

\footnotetext{
${ }^{7}$ Le rendement global, c'est-à-dire l'addition des rendements locatif et capitalistique, de l'immobilier logistique se situe en 2012 entre 7,25 et $8 \%$ (Environ $9 \%$ de rendement locatif et $-1 \%$ de rendement en capital). A titre de comparaison, le rendement global de l'immobilier de bureau ou de commerce s'établit en moyenne entre 4,5 et 7 \% (source : CBRE, 2012, JONES LANG LASALLE, 2012).

${ }^{8}$ C'est-à-dire constructeur selon la terminologie de la profession.
} 
logistique n'est plus un produit de diversification mais une affaire de spécialistes internationaux » (LA LETTRE DE LA PIERRE, 2012, p. 14). Avec ces entreprises, l'immobilier logistique tend à sortir du métier traditionnel de promoteur, et à être tiré fortement par des logiques particulières d'investisseurs, davantage internationales que les logiques de promoteurs.

Tableau 1. Les principaux investisseurs et gestionnaires de fonds d'immobilier logistique (données 2013) ${ }^{9}$

\begin{tabular}{|c|c|c|c|c|c|c|}
\hline Firmes & Nationalité & Type & $\begin{array}{l}\text { Patrimoine } \\
\text { Monde (G€) }\end{array}$ & $\begin{array}{c}\text { Patrimoine } \\
\text { Europe (G€) }\end{array}$ & $\begin{array}{l}\text { Patrimoine } \\
\text { logistique } \\
\text { (G€) }\end{array}$ & $\begin{array}{c}\text { Patrimoine } \\
\text { logistique } \\
\left(\text { millions de } \mathbf{m}^{2}\right)\end{array}$ \\
\hline Prologis & Etats-Unis & $\begin{array}{c}\text { Développeur- } \\
\text { Gestionnaire de fonds }\end{array}$ & 47 & Non connu & 47 & 55,7 \\
\hline Goodman & Australie & $\begin{array}{c}\text { Développeur- } \\
\text { Gestionnaire de fonds }\end{array}$ & 16,2 & 4,4 & 16,2 & 14 \\
\hline Segro & Royaume-Uni & $\begin{array}{c}\text { Développeur- } \\
\text { Gestionnaire de fonds }\end{array}$ & 6,6 & Non connu & 5,5 & Non connu \\
\hline Blackstone & Etats-Unis & $\begin{array}{c}\text { Investisseur- } \\
\text { Gestionnaire de fonds }\end{array}$ & 42 & Non connu & France : 0,21 & Europe : 2,5 \\
\hline GE Real Estate & Etats-Unis & Investisseur & 55 & 12,6 & 2,8 & Non connu \\
\hline Axa Reim & France & Gestionnaire de fonds & 42 & 42 & 2,5 & Non connu \\
\hline $\begin{array}{l}\text { AEW (groupe } \\
\text { BPCE) }\end{array}$ & France & Gestionnaire de fonds & 36 & 18,6 & 2 & 1,6 \\
\hline Deka & Allemagne & Investisseur & 23 & 23 & 1,4 & Non connu \\
\hline $\begin{array}{l}\text { Foncière Europe } \\
\text { Logistique } \\
\text { (Foncière des } \\
\text { régions) }\end{array}$ & France & Foncière & 1,1 & 1,1 & 1,1 & 1,8 \\
\hline WDP & Belgique & $\begin{array}{l}\text { Développeur - } \\
\text { Investisseur }\end{array}$ & 0,9 & 0,9 & 0,9 & 1,3 \\
\hline Proudreed & Royaume-Uni & Foncière & 2 & 2 & 0,8 & Non connu \\
\hline Argan & France & $\begin{array}{l}\text { Développeur - } \\
\text { Investisseur }\end{array}$ & 0,8 & 0,8 & 0,8 & 1,3 \\
\hline Vailog & Italie & $\begin{array}{l}\text { Développeur - } \\
\text { Investisseur }\end{array}$ & 0,5 & 0,5 & 0,5 & 1 \\
\hline
\end{tabular}

\footnotetext{
${ }^{9}$ Les informations sur les patrimoines correspondent aux actifs possédés ou gérés par les entreprises
} 


\begin{tabular}{|c|c|c|c|c|c|c|}
\hline $\begin{array}{c}\text { Union } \\
\text { Investment }\end{array}$ & Allemagne & Gestionnaire de fonds & 18,9 & 10,7 & 0,5 & Non connu \\
\hline $\begin{array}{c}\text { LaSalle } \\
\text { Investment } \\
\text { Management }\end{array}$ & Etats-Unis & Gestionnaire de fonds & 47 & 3,5 & 0,4 & Non connu \\
\hline $\begin{array}{c}\text { Allianz Real } \\
\text { Etate }\end{array}$ & Allemagne & Investisseur & 20 & 0,4 & Non connu \\
\hline $\begin{array}{c}\text { Appollo Global } \\
\text { Real Eastate }\end{array}$ & Etats-Unis & $\begin{array}{c}\text { Investisseur- } \\
\text { Gestionnaire de fonds }\end{array}$ & 7,2 & Non connu & Non connu & Non connu \\
\hline $\begin{array}{c}\text { CBRE Global } \\
\text { Investors }\end{array}$ & Royaume-Uni & Gestionnaire de fonds & 80 & Non connu & Non connu & Non connu \\
\hline
\end{tabular}

Sources: LA LETTRE DE LA PIERRE, 2013, sites web, rapports financiers des firmes et entretiens.

Le Tableau 1 montre que plusieurs types de firmes animent le marché de l'investissement. Deux principales catégories apparaissent: d'une part des firmes qui se cantonnent à l'investissement et d'autre part des firmes qui s'impliquent à la fois dans l'investissement et dans le développement. Figurent tout d'abord quelques foncières spécialisées dans la logistique qui développent ou acquièrent des immeubles gardés en patrimoine. De nombreuses banques et de nombreux assureurs, les investisseurs institutionnels, investissent aujourd'hui dans l'immobilier logistique de la même façon qu'ils sont à l'œuvre dans tous les types d'immobilier. Cependant, plutôt que d'acheter directement des bâtiments à la manière des foncières, ces investisseurs recourent majoritairement à des fonds d'investissement ${ }^{10}$. Apparaît donc une autre catégorie de firmes : les gestionnaires de fonds d'investissement ou gestionnaires d'actifs. Axa et AEW Europe gèrent les patrimoines logistiques les plus importants en France. Une particularité importante de l'actif logistique est sa forte présence dans les fonds d'investissement, mais très rarement dans d'autres types de montages financiers. C'est le principal vecteur de la financiarisation de l'entrepôt. C'est presqu'exclusivement par ce biais que les investisseurs institutionnels sont entrés sur ce marché. Le fonds d'investissement offre en effet des solutions particulièrement adaptées à l'immobilier logistique : abondance, flexibilité et répartition géographique. Ces modalités de la financiarisation de l'immobilier logistique ont d'importantes conséquences sur le métier de développeur.

En effet, les trois premiers développeurs mondiaux, Prologis (américain), Goodman (australien) et Segro (anglais, ancienne foncière), sont aussi des gestionnaires de fonds ${ }^{11}$. Ils proposent aux investisseurs d'entrer dans l'un de leurs fonds, certains sont cotés, d'autres non, dans lesquels ils détiennent une part variable de capital. Pour constituer ces fonds, ils développent directement l'immobilier qu'ils rachètent ensuite au titre des fonds. Transformer des entrepôts en actifs financiers logés dans des fonds d'investissement offre une capacité d'investissement (apport en capitaux des investisseurs tiers et effet de levier) à ces gestionnaires de fonds qui dépasse l'offre proposée par les promoteurs ou arbitrée par les

\footnotetext{
${ }_{10}$ Des véhicules d'investissement au sein desquels plusieurs investisseurs s'associent, en apportant chacun une part du capital, afin d'acquérir un portefeuille d'immeubles et d'en tirer un revenu à la fois par la mise en location et par la revente (arbitrage). Ces fonds sont gérés par des entreprises tierces dont l'activité consiste à sélectionner les actifs à acquérir et à revendre (l'asset management).

${ }^{11}$ Par ailleurs, existe développeur-investisseur particulier: Gazeley, filiale de Dubai World, c'est-à-dire du fonds d'investissement souverain de Dubaï.
} 
investisseurs. La mise en place de fonds d'investissement pousse ainsi ces firmes à devenir elles-mêmes développeurs et à ne pas se contenter du rôle d'investisseur. C'est pourquoi, financiarisation et intégration de la chaîne immobilière vont de pair pour le cas de la logistique.

Les entreprises dominant ce marché immobilier exercent donc à la fois les métiers de développeur, investisseur (dans ses propres fonds) et gestionnaire de fonds (pour les clients de ses fonds). De plus, dans la mesure où ces firmes ont à entretenir les bâtiments pour le compte de leurs locataires, elles exercent aussi le métier de property manager ${ }^{12}$. Enfin, non seulement l'origine de leurs capitaux est internationale, mais de plus ces firmes ont des activités de développement sur tous les continents. Avec ce type d'acteurs, l'immobilier logistique est à la fois très financiarisé et tout à fait globalisé. Ces quelques firmes intégrées gagnent en importance dans ce marché, tant en Europe qu'en Amérique du Nord et en Asie. Comment expliquer le succès de ce modèle économique, unique par rapport aux autres marchés immobiliers ? Comment parviennent-elles à être performantes à la fois en tant qu'investisseurs financiers, dans les relations avec leurs locataires et dans les négociations avec les élus locaux ?

\section{$-3-$ \\ «La montée de firmes d'infrastructures » (LORRAIN, 2002)}

La logique du développeur-gestionnaire de fonds trouve son aboutissement dans un produit immobilier particulier : le parc logistique, c'est-à-dire un complexe fermé et gardienné de plusieurs entrepôts, ayant un propriétaire (le fonds d'investissement) et gestionnaire (le gestionnaire de fonds) unique et étant directement branché sur une autoroute. Ce dernier est, sous plusieurs aspects, bien plus qu'un complexe immobilier. Il s'apparente à une infrastructure privée. Nous montrerons dans cette section que le succès du modèle économique des développeurs-gestionnaires de fonds tient justement à ce que les solutions proposées par ces firmes, tant vis-à-vis des utilisateurs d'entrepôts que des collectivités locales, s'approchent de celles de gestionnaires d'infrastructure (LORRAIN, 2002).

\subsection{Aménager des parcs logistiques}

Pour répondre à la demande de grande flexibilité spatiale des utilisateurs d'entrepôts, l'investisseur doit sécuriser des espaces au bon endroit, c'est-à-dire aux portes des métropoles. L'enjeu de sécurisation pousse l'investisseur à contrôler la chaîne immobilière et donc à devenir développeur, voire même aménageur. En effet, un projet immobilier logistique ne peut avoir lieu que sur une parcelle classée comme étant à usage d'activités économiques dans un Plan Local d'Urbanisme (PLU), c'est-à-dire située au sein d'une zone d'activités économiques existante ou à aménager. Le marché immobilier logistique est donc fortement dépendant des processus d'aménagement de ces zones. Dans la mesure où le développement des zones d'activités économiques relève de différentes réglementations et politiques mises en œuvre localement, la production d'entrepôts se trouve soumise au pouvoir des 36600 communes françaises et de leurs groupements, dont beaucoup ne sont pas en capacité

\footnotetext{
${ }^{12}$ Gestionnaires de locaux.
} 
d'aménager les grandes surfaces nécessaires aux activités logistiques. Une grande partie du travail des développeurs consiste donc à négocier avec les communes et intercommunalités des sites identifiés comme propices aux activités logistiques.

Or, un moyen de limiter cette dépendance est de transformer ces politiques locales fragmentées en un véritable marché pour les développeurs, via les concessions d'aménagement ou en étant directement lotisseur. Le développeur devient alors aménageur, c'est-à-dire qu'il prend lui-même en charge la viabilisation des sols et l'équipement en réseaux rendant les terrains constructibles. Cette intégration de la chaîne immobilière vers l'amont est cruciale parce que les logiques de l'implantation logistique, dans un contexte de flexibilité spatiale généralisée, supposent que les développeurs ou les développeursinvestisseurs prennent des positions foncières en anticipant la demande, détiennent des terrains constructibles de sorte à pouvoir développer un bâtiment aussitôt qu'un utilisateur en exprime le besoin. En effet, ces derniers, lorsqu'ils cherchent un nouveau site, ne peuvent attendre l'aboutissement d'un cycle complet depuis l'aménagement jusqu'à la livraison du bâtiment, ce qui prend entre 7 et 10 ans. De plus, les terrains mis à disposition par les aménageurs publics habituels ne suffisent pas à répondre rapidement à cette demande. L'existence d'un patrimoine foncier privé à usage logistique est une condition du développement des réseaux logistiques.

Cette logique trouve son aboutissement dans un produit immobilier particulier : le parc logistique. L'enjeu de la sécurisation du foncier pousse les investisseurs à aménager et posséder des parcs logistiques entiers en lieu et place d'entrepôts isolés au sein de zones d'activités aménagées et gérées par des collectivités locales. En effet, ces derniers sont souvent menacés lorsqu'ils sont inoccupés et sont à la merci d'un changement de vocation de la zone d'activités, sur lequel l'investisseur n'a pas de prise. De plus ce type de gestion ne permet pas d'offrir le service immobilier flexible demandé. En contrepoint, le parc logistique apporte une double solution. Il sécurise ces espaces pour les activités logistiques dans la mesure où il consiste en la privatisation de la totalité d'une zone d'activités. L'investisseur peut aussi plus facilement faire évoluer son service aux besoins de ses clients : proposer une surface plus petite ou plus grande si nécessaire. Dans le même temps, le parc logistique fixe durablement une fonction au territoire concerné. La logistique devient en quelque sorte la vocation de ce territoire et entre plus nettement dans la stratégie économique de ce dernier. Cela limite le risque de mutation de la fonction de la zone. Comme l'investisseur de parc logistique est aussi property manager, la firme en vient ainsi à intégrer la totalité de la chaîne immobilière de l'aménagement à la gestion immobilière. Prologis, numéro un mondial de l'immobilier logistique, est la firme qui a poussé le plus loin cette logique (RAIMBAULT, 2013b).

C'est pourquoi la dynamique d'intégration de la chaîne immobilière vers l'aménagement et la multiplication du nombre de parcs logistiques sont deux facettes du succès du modèle économique du gestionnaire d'infrastructure. Ce modèle économique a d'importants impacts sur l'action publique puisqu'il suppose une privatisation à la fois de l'aménagement (sous forme de lotissement ou de concession) et de la gestion à long terme de la zone logistique (les espaces publics et le choix des entreprises qui s'implantent). L'aménagement de parcs logistiques promeut ainsi les firmes de l'immobilier logistique au rang d'acteurs de l'action publique locale.

\subsection{Le modèle du gestionnaire d'infrastructure}

En aménageant des parcs logistiques, les aménageurs-développeurs et gestionnaires de fonds se rapprochent du modèle du gestionnaire d'infrastructure, s'opposant à celui du 
promoteur. Ce modèle est présenté de la façon suivante par LORRAIN (2002, p. 28). Les capitaux sont plus importants à mobiliser (un parc logistique représente un investissement d'environ 200-300 millions $€$ ), l'horizon temporel est plus long (la durée de vie projetée d'un parc logistique est de 30 ans), les relations sont plus suivies avec la puissance publique que dans le cas de la promotion d'un simple bâtiment.

Le parc devient une quasi-infrastructure selon deux principales modalités. D'une part, en devenant un équipement nodal fixe standardisé dans un vaste réseau logistique flexible et mouvant, le parc logistique se rapproche de la définition de l'infrastructure de CURIEN (1993). D'autre part et surtout, le parc logistique s'ancre dans un territoire au point de représenter une quasi fonction d'intérêt général localement reconnue. En effet, il suppose un partenariat entre le développeur et la collectivité. Même s'il s'agit d'un projet privé, la taille et la visibilité d'un parc logistique nécessite un soutien de la part de la commune qui doit le porter politiquement, adapter son PLU et donc produire un projet territorial. Cette logique est renforcée lorsque le gestionnaire de parc procède lui-même à l'aménagement. La coopération avec la commune est nécessairement plus longue et suivie. Plus encore, le parc logistique est une façon non juridique ${ }^{13}$ de faire reconnaître localement l'utilité de l'équipement à cet endroit. La collectivité locale porte non seulement le projet d'une zone d'activités économiques mais clairement et spécifiquement le projet d'une zone uniquement logistique, ayant vocation à le rester au moins 30 ans. La spécialisation logistique de l'équipement est donc reconnue comme correspondant à l'intérêt de la collectivité locale. Le gestionnaire de parc jouit ainsi d'une forme de délégation de service public implicite. La collectivité locale porte politiquement le projet mais son développement et sa gestion sont laissés à l'investisseur privé qui lui s'engage dans un processus de coopération. Ces entreprises ne peuvent donc rester hors-sol à la manière de l'investisseur purement financier. En intervenant à toutes les étapes de la chaîne de valeur, elles sont de fait en relation à la fois avec les entreprises et les collectivités territoriales. Ces firmes sont des propriétaires locaux ayant pignon sur rue. Leurs entrepôts sont, par exemple, signés «Goodman » ou «Prologis ». Autrement dit, à côté des investisseurs surplombants et leur «main discrète », imposant leurs critères à la production urbaine (LORRAIN, 2011a), existent dans l'immobilier logistique des firmes à la fois investisseurs et opérateurs jouant de «leur main visible » pour que la production et la gestion des zones logistiques leur offrent les rendements escomptés.

Plusieurs paradoxes apparents découlent de cette analyse du modèle économique des gestionnaires d'infrastructure. Ce dernier suppose une privatisation d'une partie des politiques locales d'aménagement et de développement économique tout en rendant incontournable la coopération entre l'investisseur immobilier et la collectivité locale. Dans le même mouvement, il privatise et politise la production des espaces logistiques. De plus, dans la mesure où ce modèle économique est essentiellement mis en œuvre par des entreprises internationales, les acteurs qui s'ancrent localement, tissent des partenariats et s'inscrivent le plus avant dans le long terme de l'infrastructure sont des firmes globales. L'intégration de la chaîne immobilière recouvre ainsi l'enjeu de l'intégration des échelles du global au local. Il s'agit d'un côté de négocier avec des utilisateurs et des investisseurs et de l'autre de négocier avec le politique et les propriétaires fonciers locaux. Cela nécessite donc d'intégrer deux échelles : celle des investisseurs et celle des clients, nationale voire globale, et celle de la production du foncier et de la négociation politique, qui est locale. Qu'est-ce qui explique le succès local des firmes internationales ?

\footnotetext{
${ }^{13}$ Le parc n'apparaît pas comme zone logistique dans le Plan Local d'Urbanisme (PLU) de la commune mais seulement comme zone d'activités économiques.
} 


\subsection{L’intégration des échelles du global au local}

Le défi des développeurs-gestionnaires de fonds internationaux est de répondre à la problématique de la flexibilité immobilière nécessaire aux utilisateurs d'entrepôts. Comme cette dernière est peu ou prou globale, la problématique immobilière se pose à cette échelle. Pour suivre ses clients, l'investisseur tend à constituer le patrimoine le plus important possible à l'échelle mondiale et ainsi à offrir plus de flexibilité aux clients, c'est-à-dire un choix d'implantations plus grand. À l'échelle européenne, le seuil jugé nécessaire pour pouvoir jouer de cet effet taille est estimé à $1 \mathrm{G} €$ de patrimoine, soit entre 3 et 5 millions de $\mathrm{m}^{2^{14}}$. Prologis en est le symbole. Il propose ainsi 55 millions de $\mathrm{m}^{2}$ d'entrepôts sur les quatre continents à enjeux logistiques: Amérique du Nord et du Sud, Europe, Extrême-Orient. Il « est capable de dire aujourd'hui : on vous déchire un bail à un endroit et on vous retrouve des surfaces ailleurs » (Entretien Direction Prologis : 14/09/2011). De plus, les standards vont vers des entrepôts voire des parcs de plus en plus grands et donc vers des coûts d'investissement de plus en plus importants, ce qui constitue une barrière supplémentaire à l'entrée de ce marché, c'est-à-dire un facteur supplémentaire expliquant à la fois la concentration et la présence d'acteurs internationaux ayant accès aux capitaux.

Mais si l'activité est globale, les modalités de production de l'espace logistique sont locales. Comment le développeur-investisseur mondial parvient-il à articuler les deux échelles ? En raison de l'activité de gestion immobilière des parcs, le travail de ces entreprises se décline aussi localement. Chaque ensemble est géré par son property manager, qui est régulièrement présent dans le site et en contact avec les mairies. Ainsi, lorsque l'investisseur s'installe durablement dans le territoire dans le cadre de la gestion d'un parc logistique bien visible, avec un horizon de plusieurs dizaines d'années, il s'apparente, bien qu'incomplètement, à une firme «locale-globale ». C'est le pendant du modèle du gestionnaire d'infrastructure présenté plus haut lorsqu'il est jumelé à une activité globale. L'échelle locale n'est pas aussi importante que pour l'opérateur de service urbain, le développement ne passant pas par des contrats d'exploitation à aussi forte connotation politique (LORRAIN, 2002, p. 24). Cependant, le développeur-investisseur doit justifier localement sa présence, être supporté politiquement, rendre des comptes, faire évoluer si besoin son actif pour qu'il réponde aux critères du marché et à la demande locale, parfois politique, de développement économique.

Au-delà de la gestion locative, l'enjeu de l'articulation au local se pose principalement dans la relation aux gouvernements locaux, tout particulièrement lors du développement des parcs logistiques puis, de manière plus diffuse, durant les trente années de gestion de ce parc. De même que pour tous les projets immobiliers, "un processus de commutation est à l'œuvre » pour le développement logistique.

"L'idée de commutation renvoie explicitement à un processus de territorialisation de la mondialisation. L'ancrage ou " atterrissage " des capitaux internationaux fait concrètement l'objet d'une " médiation " par un système sociotechnique régional qui, loin d'être seulement un réceptacle, contribue également à façonner ces flux » (DAVID et HALBERT, 2010, p. 96).

Quelles en sont les modalités pour l'immobilier logistique ? Ces dernières expliquent-telles en partie la forme particulière de ce marché et le succès des firmes intégrées ? Le marché immobilier résidentiel, mais aussi, bien que dans une moindre mesure, l'immobilier commercial et de bureau, restent dans la pratique particulièrement encastrés dans l'action

\footnotetext{
${ }^{14}$ Source : BuSINESS IMMO, 2013.
} 
publique, notamment locale. Le politique conserve sur lui un pouvoir encore déterminant. Le contact avec les élus, et notamment le maire détenant les principaux pouvoirs d'accès au foncier, est essentiel au bon déroulement du projet (POLLARD, 2009). Cet encastrement est invoqué pour expliquer la faible internationalisation des promoteurs immobiliers et le rôle incontournable des «acteurs-ancreurs » (THEURILLAT, 2011). Au contraire, l'immobilier logistique y échappe-t-il partiellement ?

\section{$-4-$ \\ La privatisation des politiques d'aménagement et de développement économiques des territoires périurbains}

L'argument du moindre encastrement local mérite d'être pris en compte dans la mesure où le modèle économique du parc logistique suppose une privatisation des politiques d'aménagement et de gestion des zones d'activités économiques. Il implique donc que les collectivités locales se dessaisissent d'une partie de leurs pouvoirs. Autrement dit, les modalités de la financiarisation de l'immobilier logistique supposent d'une part que les collectivités locales concernées soient favorables à ce type d'implantation économique et, d'autre part, qu'elles soient disposées à déléguer la conception et la mise en œuvre de l'aménagement d'une zone d'activités puis de sa gestion à un aménageur-développeur privé, c'est-à-dire que cette privatisation réponde à une demande locale.

Le développement d'un parc logistique suppose ainsi un alignement des acteurs publics et privés, réunis autour d'un intérêt commun au développement logistique, ce qui correspond à la notion de «coalition de croissance» de LOGAN et MoLOTCH (1987), ici selon une déclinaison logistique. Cet alignement rend compte d'une forte interdépendance entre les acteurs privés et publics. Or celle-ci est au fondement de l'approche des régimes urbains (STONE, 1993, p. 2) considérant que les politiques urbaines sont élaborées par des coalitions informelles, mais stables, unissant des groupes d'acteurs porteurs de ressources et d'intérêts variés. Cette approche constitue en ce sens un cadre d'analyse particulièrement pertinent pour appréhender les liens entre action publique locale et modalités de la financiarisation de l'immobilier logistique.

De plus, PHELPS montre que les territoires périurbains constituent un contexte favorable à l'émergence de coalitions de croissance ou de régimes urbains davantage que les villes centres (PhelPs et al, 2006 ; PhelPS et WoOd, 2011 ; PhelPS, 2012). Ces conclusions se rapprochent de celles des travaux mettant en évidence la montée en puissance d'un «urban privatism» (BARNEKOV et al., 1989) et, par conséquent, d'une «post-political » ou «post-democratic city » (SWyngedouw, 2009 ; MaCLEOD, 2011): des pans de plus en plus importants de l'action publique locale seraient privatisés et mis au service des intérêts des élites économiques par le truchement de différentes formes de partenariats publics privés, du remplacement progressif des gouvernements élus par des agences, de la fermeture et la sécession de certaines territoires urbains (GRAHAM et MARVIN, 2001 ; MACLEOD, 2011) et plus largement du consensus en faveur de la croissance économique (HARVEY, 1989). La gouvernance des territoires du développement logistique est-elle, en ce sens, pilotée voire privatisée par les firmes de l'immobilier logistique au service de leurs seuls intérêts ? La réponse à cette question suppose de caractériser le pouvoir des firmes de l'immobilier logistique à la fois selon une approche fonctionnaliste, le «pouvoir pour » développer des 
parcs logistiques, et selon une approche distributive, le «pouvoir sur » les collectivités locales, selon le cadre analytique proposé par NiCHOLLS (2005).

\subsection{Un environnement favorable}

Une approche statistique et cartographique du développement logistique de la métropole parisienne ${ }^{15}$ met en évidence un mouvement de «périurbanisation logistique » original et assez différent de celui imprimé par la construction résidentielle et le desserrement des autres activités économiques (BAHOKEN et RAIMBAULT, 2012). Le développement logistique a majoritairement lieu dans certains espaces situés en périphérie, voire en grande périphérie, des métropoles. Plus précisément, les communes correspondant au développement de grands parcs logistiques sont particulièrement petites et périphériques. Elles ont en moyenne moins de 5000 habitants selon un spectre allant de 200 à 26000 habitants. Elles sont généralement situées en dehors des intercommunalités puissantes (RAIMBAULT et BAHOKEN, 2014). Pour ces raisons, ces collectivités sont généralement dotées d'une faible capacité administrative, technique et financière. Elles possèderaient donc rarement toutes les ressources administratives, opérationnelles et financières nécessaires pour conduire les projets d'aménagement d'envergure que sont les zones logistiques. De plus, en termes de développement économique, leur attractivité est limitée. Si elles décident de mettre en œuvre une politique de développement économique, elles n'ont généralement guère d'autres choix que d'accueillir des activités logistiques. Cette situation s'est encore renforcée depuis la crise financière de 2008.

Finalement, nous posons l'hypothèse que la géographie de plus en plus périphérique des projets logistiques offrirait aux développeurs-investisseurs des mécanismes de « commutation locale », un environnement de régulations publiques, plus favorables que dans des espaces plus centraux. Dans ces «franges [de la métropole] où la densité institutionnelle (...) est plus molle »(LORRAIN, 2011b, p. 25) que dans les cœurs métropolitains, des acteurs stratèges globaux peuvent mener des opérations, tirant profit de l'asymétrie de pouvoir en leur faveur. Ces espaces politiques seraient caractérisés par un vide politique exploité par les acteurs de l'immobilier logistique, ce qui rejoint la thèse de STONE à propos des régimes urbains : "The weakness of formal authority leaves a vacuum that business interest have the ready resources to fill » (STONE, 1993, p. 26).

\subsection{Des coalitions pour construire une capacité à développer des zones logistiques : l'exemple du Val Bréon}

La Communauté de communes du Val Bréon (CCVB) est située à environ $50 \mathrm{~km}$ à l'est de Paris, dans sa couronne périurbaine. Elle est peuplée d'environ 15000 habitants répartis en 10 communes. Une grande zone de 200 ha dédiée à la logistique, rassemblant $400000 \mathrm{~m}^{2}$ d'entrepôts, y a été aménagée sur le territoire communal de Châtres, peuplée d'à peine 600 habitants. Le projet immobilier est donc de très grande ampleur. Il représente un investissement de 300 millions $€$. Il fait contraste avec la taille des communes concernées et leur situation très périphérique. Dans la mesure où ces dernières n'appartiennent pas à une intercommunalité puissante, leurs capacités administratives sont limitées. Elles détiennent

\footnotetext{
${ }^{15}$ Définie comme l'Île-de-France et ses départements limitrophes.
} 
cependant les ressources légales indispensables à la réalisation du projet. Quelle coalition le développement de ce parc logistique a-t-il nécessité ?

La mise à l'agenda du parc logistique est publique. Il s'agit d'un projet communal devenu rapidement intercommunal. La CCVB a été créée en 1995 pour porter ce parc, le secteur logistique apparaissant comme particulièrement porteur aux yeux des élus. Leur principale motivation est de créer ainsi des ressources fiscales permettant de mettre en œuvre de nouvelles politiques publiques intercommunales : transport à la demande et portage de repas à domicile. Cependant la communauté de communes n'a pas les capacités administratives, juridiques et financières de développer un tel projet.

"Elle ne souhaitait pas la porter juridiquement, financièrement. (...) Il était hors de question d'y mettre un centime et donc ça avait vocation à devenir une opération complètement privée. (...) Elle n'a pas les moyens, ni financiers, ni les compétences techniques." (Entretien aménageur-développeur : 14/10/2011)

Les élus se mettent en recherche d'un aménageur-développeur pour financer et conduire ce qui représente alors la plus grande Zone d'Aménagement Concerté (ZAC) privée d'Île-deFrance. Le développeur qui décroche la concession d'aménagement doit financer la totalité de l'opération (300 millions $€$ ), gérer l'aménagement puis la construction et la vente des 400000 $\mathrm{m}^{2}$ de bâtiments logistiques construits. La collectivité ne tient pas à peser sur le choix des entreprises. Cependant, l'histoire de ce projet va au-delà de celle d'une concession. Le rôle de l'aménageur privé s'étend au pilotage du projet, y compris dans ses dimensions politiques. Le principal problème qui se pose est de négocier une sortie de crise avec une association de défense de l'environnement qui a posé plusieurs recours. Les relations étant rompues entre l'association et les élus locaux, c'est l'acteur privé qui hérite de cette question pourtant hautement politique. A l'issue d'une négociation conduisant à associer les écologistes au projet d'aménagement, c'est-à-dire à les faire rentrer dans la coalition, un arrangement quasiment privée est trouvée sous les conseils du développeur : des terrains sont cédés par l'aménageur à l'association pour $1 €$ symbolique et des servitudes (de droit privé) de nonconstructibilité sont édictées. Par ailleurs, la société s'investit largement dans la révision du PLU de Châtres. L'acteur privé a ainsi mené nombre d'actions habituellement conduites par les collectivités locales dans le cadre de leurs compétences d'urbanisme et de développement économique. En avril 2009, tous les bâtiments sont construits et vendus à divers investisseurs partenaires. Ils sont rapidement tous occupés par des grands noms de la logistique et emploient maintenant près de 1000 personnes.

Les exigences publiques se sont essentiellement limitées à «la qualité paysagère générale » (Entretien Président CCVB: 15/10/2011) présentée comme une démarche de développement durable. La collectivité a finalement abandonné une certaine maîtrise de son territoire en échange d'économies considérables. Elle a pris en charge moins de $10 \%$ de l'investissement alors que les recettes fiscales générées par la zone logistique financent presque totalement le budget de 10 millions $€$ de la CCVB.

L'incapacité et la relative faiblesse des objectifs publics locaux ont donc offert l'occasion au développeur immobilier de privatiser de manière poussée la politique de développement économique locale. Comme nous l'avons déjà esquissé, c'est un enjeu cher aux investisseurs immobiliers. La relation entre le développeur et la CCVB peut être décrite comme une coalition de croissance puisque l'entreprise privée a réalisé la plus-value foncière grâce à l'action de la collectivité publique, qui en retire un bénéfice fiscal. Mais l'origine du projet est bien publique: la privatisation résulte d'une demande d'action privée exprimée par la collectivité. La coalition a été forgée de sorte à avoir les capacités locales pour développer la zone logistique, c'est-à-dire une capacité à gouverner, soit la finalité d'un régime. La 
collectivité apporte ses pouvoirs légaux, le développeur privé apporte toutes les autres ressources nécessaires.

Il apparait à travers cette étude de cas que les territoires périphériques tels que Val Bréon correspondent à des environnements régulatoires favorables à de tels développeurs. Le «vacuum » est une ressource exploitée par les acteurs de l'immobilier. Le pouvoir de l'acteur immobilier trouve sa légitimité dans le fait que cette privatisation apporte une solution aux problèmes que se posent ces collectivités locales : une capacité à aménager une zone logistique répondant aux critères des utilisateurs d'entrepôts, ce qu'elles ne sont pas à même de construire seules. Le cas du Val Bréon montre que des coalitions se forment entre (communautés de) commune(s) et développeurs-investisseurs afin de construire une capacité à développer des zones logistiques dans des territoires qui en sont dépourvus, c'est-à-dire afin de construire un pouvoir pour le développement. La géographie particulièrement périphérique du développement logistique est donc un facteur favorable au modèle économique du parc logistique, elle simplifie les enjeux de la commutation locale pour ces acteurs internationaux. Réciproquement, les firmes de l'immobilier logistique, étant à la recherche de fonciers facilement exploitables, et donc périphériques, accentuent cette périurbanisation logistique.

\subsection{Une privatisation au nom de l'intérêt général : le cas du parc Prologis de Moissy- Cramayel à Sénart}

Le rôle des firmes de l'immobilier logistique ne se limite pas au temps du développement, que nous venons d'analyser avec le cas du Val Bréon. La durée d'amortissement d'un parc logistique est de trente ans. Par conséquent, pendant trente ans, le gestionnaire de parc a le pouvoir de choisir les entreprises s'implantant dans la commune ${ }^{16}$, leur profil, le nombre et le type d'emplois au sein du parc logistique. Le gestionnaire gère aussi souvent le transport des salariés et des services annexes tels que des crèches et des restaurants inter-entreprises. Les parcs logistiques se rapprochent ainsi des « "briques » juridiquement autonomes et souvent morphologiquement séparées » mises en évidence par LORRAIN (2011a) comme l'un des impacts matériels de la finance globale sur la production urbaine. Ils correspondent plus largement à la notion d' «urban privatism » que l'on peut définir comme «an underlying confidence in the capacity of the private sector to create the conditions for personal and community prosperity » (BARNEKOV et al, 1989, p. vii), c'est-à-dire, en ce qui nous concerne, l'idée que les acteurs privés seraient les plus pertinents (efficaces et légitimes) pour développer et gérer ces infrastructures, ces services locaux aux activités économiques. Or, comme nous l'avons expliqué, cette privatisation suppose le soutien de la commune concernée. Pourquoi le modèle du parc logistique emporte-t-il l'adhésion comme mode de gestion des espaces logistiques, au-delà de la question de la capacité à produire des espaces logistiques?

Le cas du parc logistique Prologis de Moissy-Cramayel, situé dans la ville nouvelle de Sénart, est particulièrement intéressant dans la mesure où une ville nouvelle correspond à un contexte institutionnel très différent de celui du Val Bréon. Les capacités publiques locales y sont très développées. En effet, la ville nouvelle étant encore entièrement sous statut d'Opération d'Intérêt National (OIN), les politiques d'aménagement et de développement économique sont directement aux mains de l'Etat via l'Etablissement Public d'Aménagement (EPA) de Sénart qui est sous sa tutelle. La présence de l'EPA offre à la ville nouvelle d'importantes capacités locales pour développer des zones logistiques et attirer des

\footnotetext{
${ }^{16}$ Le parc logistique constitue souvent la seule zone d'activités économiques de la commune concernée.
} 
établissements logistiques en nombre. Dans ce but, l'établissement a tissé des liens très étroits avec un certain nombre de promoteurs et d'investisseurs de l'immobilier logistique, plutôt nationaux, qu'il organisa peu à peu en réseau. Cependant, il n'a pas impulsé le développement de parcs logistiques privés, dans la mesure où son métier, et donc son chiffre d'affaires, consiste justement en l'aménagement et la commercialisation des terrains. Grâce à l'action de l'EPA, le développement logistique est au rendez-vous. La ville nouvelle de Sénart constitue l'un des tous premiers pôles logistiques régionaux. Ce secteur représenterait aujourd'hui 7.000 emplois, soit près de $20 \%$ de l'emploi local, et se traduirait par plus de 1,8 million $\mathrm{m}^{2}$ d'entrepôts ${ }^{17}$.

Le réseau d'immobilier logistique monté par l'EPA est cependant peu à peu remis en cause à mesure que les communes cherchent à davantage contrôler leur développement. Une défiance s'est installée. Ce nouveau contexte offre cependant l'opportunité de développer différemment les projets logistiques, opportunité tout particulièrement exploitée par Prologis et sa stratégie de développement de parcs logistiques. Cette firme ne fait pas partie du réseau de l'EPA. Elle parvient cependant à développer dans une des communes de Sénart, à MoissyCramayel, un parc logistique, un deuxième étant même en cours d'aménagement sur cette même commune. L'irruption de cette firme, et la privatisation de la politique d'aménagement et de développement économique qui s'ensuit, rencontre paradoxalement certaines exigences locales en matière de gestion de ces zones, c'est-à-dire la recherche d'une plus grande gouvernabilité du développement logistique.

Au début des années 2000, Prologis acquiert à Moissy-Cramayel une centaine d'hectares auprès d'un agriculteur dont l'exploitation est en liquidation judiciaire. Il s'ensuit une longue négociation avec la mairie pour changer le PLU de telle sorte que Prologis puisse développer de manière privée un parc logistique. Trois arguments en particulier convainquent le maire, après la visite d'un parc Prologis en Angleterre : la capacité à intégrer le parc dans le paysage, le fait que ce dernier soit clos, gardienné avec des services de sécurité, et que sa gestion, c'està-dire l'entretien de la voirie et des espaces verts, soit prise en charge, techniquement et financièrement, par Prologis. En un mot, c'est le modèle du parc logistique, comparé à celui des zones d'activités économiques aménagées et gérées par l'EPA, qui emporte son adhésion. Cependant, la municipalité s'est trouvée de ce fait en opposition frontale avec l'EPA, qui, pour la première fois, était débordé dans son rôle de planification et dans celui d'aménageur et de commercialisateur, et donc aussi de prescripteur des prix du foncier dans la zone. Ce projet a donc nécessité la construction d'une coalition de croissance montée contre le régime immobilier particulier de l'EPA Sénart.

"Avec le maire de Moissy ça a été à un moment donné une histoire de confiance. (...) Une histoire d'hommes. » (Entretien Direction Prologis : 14/09/2011)

Le modèle économique de Prologis a en effet rencontré l'intérêt municipal sur plusieurs points : financier, mais aussi politique. Il est apparu comme une solution de production et de gestion des espaces logistiques localement avantageuse, plus avantageuse que celle proposée par l'EPA. Il a d'abord été soutenu dans la mesure où à la fois l'investissement et les frais de gestion sont assumés par l'acteur privé, à l'inverse des zones d'activités aménagées par l'EPA qui reviennent à la charge des communes, c'est-à-dire qui pèsent sur les ressources locales. Mais peut-être plus encore que la dimension financière, c'est le gain de pouvoir local paradoxal ouvert par la privatisation de l'aménagement qui a décidé le maire de Moissy-

\footnotetext{
${ }^{17}$ Source: Contrat de Développement Territorial de Sénart, décembre 2013. Si l'on tient compte de tous les emplois logistiques, y compris ceux que les industriels et la grande distribution n'ont pas externalisés, la proportion pourrait même être double, soit $40 \%$ de l'emploi local.
} 
Cramayel. En effet, si l'EPA est en capacité de développer des zones logistiques de manière largement autonome, tel n'est pas le cas des développeurs privés :

"J'avais en face de moi des investisseurs privés qui étaient totalement tributaires de l'accord des élus, donc ils ont été attentifs à l'attente des élus. C'est vrai que l'établissement public n'a pas complètement besoin de moi pour faire, donc ils sont peut-être moins attentifs à la demande des élus. »(Entretien Maire : 17/06/2011)

Ce gain de pouvoir rencontre la revendication de contrôle territorial exprimée par les communes contre l'EPA, c'est-à-dire de plus grande gouvernabilité locale des espaces logistiques.

Au-delà de la seule production du parc, c'est aussi en tant que mode de gestion sur le long terme que la solution proposée par Prologis est localement soutenue, soit le modèle du gestionnaire d'infrastructure. L'usage du parc clarifie à la fois la vocation de la zone et sa gestion: elle est dédiée à la logistique, c'est Prologis qui en est le propriétaire ${ }^{18}$ et le responsable. L'interlocuteur est ainsi clairement identifié. C'est un interlocuteur durable qui s'engage à gérer son parc sur le temps long (30 ans). Il devient un acteur de la commune, localement présent, à qui l'on peut demander des comptes. Ce sentiment de gain de pouvoir est paradoxal puisque la solution de Prologis consiste justement en une délégation complète des pouvoirs d'aménagement et de gestion d'une zone, pouvoirs qu'une commune ou un syndicat intercommunal ont sur les autres zones.

De son côté, Prologis se sert de cette réalisation comme d'une vitrine, d'un modèle parmi ses réalisations. Le nom de Prologis et celui de Moissy-Cramayel s'associe durablement, son maire devient un ambassadeur des «solutions aux collectivités » proposées par l'entreprise. Le maire de Moissy-Cramayel «est un super ambassadeur pour nous. Sur le site de Moissy, on avait au moins une visite d'un député, ou d'un maire d'une commune française toutes les semaines » (Entretien Direction Prologis : 14/09/2011).

Nouvel indice des capacités de développement de cette coalition, Prologis a acquis en 2012 le site de PSA (situé à Moissy-Cramayel) de 60 hectares afin d'y développer un nouveau parc logistique de $230000 \mathrm{~m}^{2}$ pour un investissement estimé à 200 millions $€$. Une fois de plus, Prologis a trouvé le moyen de s'implanter à Sénart sans recourir à l'EPA : l'acquisition du site est un processus privé, la mise en œuvre du projet ne nécessite que des modifications de PLU. À terme, Prologis aura ainsi développé $490000 \mathrm{~m}^{2}$ d'entrepôts, soit plus d'un quart du parc total de Sénart, sans jamais acheter un $\mathrm{m}^{2}$ de terrain à l'EPA.

Ces études de cas montrent combien les institutions, les intérêts et les représentations des acteurs publics locaux, c'est-à-dire les modalités de la réception locale des projets immobiliers logistiques, sont un élément d'explication central du fonctionnement du marché immobilier logistique et des modalités de sa financiarisation. Le modèle du développeurgestionnaire de fonds et de parcs logistiques suppose que la privatisation des politiques d'aménagement et de développement économique soit localement soutenue et corresponde à la représentation que ces collectivités se font de leur intérêt général. Cette thèse peut être illustrée en creux par l'exemple des Pays-Bas. "What is different in the Netherlands compared to France is that we have municipalities which are only selling land per parcel. So we can only buy a land for one warehouse. And the road in front is still owned by the municipality. So we have to do case by case and because of that, you get a warehouse here, a warehouse there but you can't develop a park» (Entretien direction Prologis Benelux : 04/03/2013). Dans ce pays, le développement logistique n'a pas lieu dans de petites

\footnotetext{
${ }^{18}$ Même s'il n'est propriétaire que d'une part du fond d'investissement où est logé le parc.
} 
communes mais dans des villes ayant une administration développée, soutenue par une longue tradition de prise en charge du processus d'aménagement et de contrôle de la rente foncière. En raison de cette maîtrise publique, Prologis n'a pas la possibilité d'y développer des parcs logistiques (RAIMBAULT et al, 2015). En ce sens, les règles locales, et surtout leur mise en œuvre, déterminent fortement le modèle économique que Prologis développe dans tel ou tel marché. La configuration locale française est plus proche de celle que connait Prologis aux Etats-Unis où l'entreprise a forgé son modèle de parcs logistiques. Par conséquent, dans ces espaces où les capacités des gouvernements locaux sont peu développées, parfois en cours de développement sous la forme de nouvelles intercommunalités comme dans le cas du Val Bréon, le modèle économique des firmes de l'immobilier logistique catalyse des arrangements de gouvernance particuliers, contribue à définir les règles du jeu de l'action publique locale, à définir ce qui relève du champ de l'action publique et de ce qui n'en relève pas.

\section{$-5-$ \\ Conclusion}

La financiarisation de l'immobilier logistique prend une forme particulière via l'activité des développeurs-gestionnaires de fonds globaux, c'est-à-dire via un mouvement liant globalisation, intégration de la chaîne immobilière et ancrage local. Cette spécificité s'explique d'une part par les particularités de la demande logistique (géographie périphérique, baux courts, standardisation des process logistiques) et d'autre part par les modalités de la réception locale des projets immobiliers logistiques. Cette situation offre à ces acteurs un pouvoir considérable. Ils sont à même d'apporter une capacité à développer des zones logistiques et, par là, à attirer des établissements logistiques dans des territoires dont les gouvernements locaux sont dépourvus de telles capacités, c'est-à-dire un « pouvoir pour » le développement de nouvelles zones logistiques. En d'autres termes, l'industrie immobilière planifie les investissements et les répartie dans l'espace, les communes reçoivent ces projets en accommandant plus ou moins leurs territoires aux critères des acteurs immobiliers. De plus ce «pouvoir pour» s'exerce par un «pouvoir sur» ces collectivités locales. Ce mode de production des espaces logistiques conduit à privatiser un certain nombre de politiques publiques locales. En ce sens, l'analyse du modèle économique des grandes firmes de l'immobilier logistique montre que, si elles sont contraintes de s'ancrer localement pour y mener leur activité, elles sont parvenues à transformer cette contrainte en levier de pouvoir. En intégrant la chaîne jusqu'à l'aménagement et donc en s'ancrant davantage, ces firmes sont devenues des acteurs des politiques publiques locales d'aménagement et de développement économique au point d'être à même de changer les règles en leur faveur, tout en légitimant ces privatisations comme relevant de l'intérêt général local.

Cette privatisation, si elle répond aux problèmes publics construits localement, pose des problèmes cruciaux de régulation publique. Avec le parc logistique privé, l'action publique est largement aveugle aux enjeux de production des services logistiques, qu'il s'agisse du report modal ou d'emploi : conditions de travail, adéquation avec le marché du travail local, déplacements domicile-travail. En effet, les acteurs publics locaux n'ont, dans ce cadre, plus de relations avec les utilisateurs d'entrepôts, les entreprises et les salariés qui produisent les services logistiques, puisque ces derniers sont uniquement représentés par le gestionnaire du parc. Le monde de la logistique est donc appréhendé par le seul prisme du gestionnaire immobilier qui lui seul a accès à ses locataires et les choisit. La logistique devient une boîte noire pour les acteurs publics locaux. 
Finalement, en complément d'une analyse de la financiarisation de la production urbaine à travers la commutation du capital vers les territoires, l'étude précise de l'action publique, notamment à travers les coalitions public/privé, permet de montrer dans quelle mesure les investisseurs immobiliers pèsent sur les politiques locales, s'y adaptent et les redéfinissent, c'est-à-dire font de la politique (GUIRONNET et HALBERT, 2014). Les modalités de la financiarisation de l'immobilier logistique, et la dynamique de privatisation qu'elles enclenchent, mettent ainsi en évidence l'existence de marges au sein de l'action publique, des «governance discontinuities » (BORRAZ et LE GALES, 2010, p. 9) au sein de la métropole.

\section{Remerciements}

Cet article a été écrit pour puis présenté à la session spéciale «finance de marché et territoires » du colloque 2014 de l'Association de Science Régionale de Langue Française (ASRDLF). Nous remercions chaleureusement les organisateurs de cette session, Olivier Crevoisier, Ludovic Halbert et Thierry Theurillat, ainsi que tous les participants, pour les riches échanges qu'elle a suscités.

\section{Références bibliographiques}

BAHOKEN F, RAIMBAULT N (2012) La périurbanisation singulière de l'immobilier logistique du Bassin parisien. M@ppemonde 106 [en ligne] http://mappemonde.mgm.fr/num34/articles/art12202.html

Barnekov T, Boyle R, Rich D (1989) Privatism and Urban Policy in Britain and the United States. Oxford University Press.

BorRRAZ O, LE GALÈs P (2010) Urban Governance in Europe: the Government of What? Métropoles 7 [en ligne] http://metropoles.revues.org/4297

Bretagnolle M (2012) Plate-forme d'un secteur clef. L'entrepôt logistique évolue. Mémoire de DESS. Cycle d'urbanisme de Sciences-Po, Paris.

CURIEN N (1993) Économie des réseaux. La découverte, Paris.

Dablanc L, Fremont A (dir.) (2015) La métropole logistique. Armand Colin, Paris.

DAVID L, HALBERT L (2010) Logiques financières globales et fabrique de la ville. In: JACQuet P, PAChauri R K, TUbiana L (dir.) Regards sur la Terre. Presses de Sciences Po, Paris.

DAVID L, HALBERT L (2013) Constructing 'world-class' cities: hubs of globalization and high finance. Dialogues in Urban and Regional Planning 5: 99-114.

DAVID L, HALBERT L (2014) Finance capital, actor-network theory and the struggle over calculative agencies in the business property markets of Mexico City Metropolitan Region. Regional Studies 48 (3): 516-529.

Guironnet A, HALBERT L (2014) The Financialization of Urban Development Projects: Concepts, Processes, and Implications. Document de Travail Du LATTS - Working Paper [en ligne] https://hal-enpc.archives-ouvertes.fr/hal-01097192

Guironnet A, Attuyer K, Halbert L (2015) Building Cities on Financial Assets: The Financialisation of Property Markets and Its Implications for City Governments in the Paris City-Region. Urban Studies $\quad$ [en ligne] http://usj.sagepub.com/content/early/2015/04/02/0042098015576474.abstract 
GraHAM S, MARVIN S (2001) Splintering Urbanism: Networked Infrastructures, Technological Mobilities and the Urban Condition. Blackwell, Oxford.

GRANELLE J-J (1998) Économie immobilière : analyses et applications. Économica, Paris.

HALBERT L (2013) Les acteurs des marchés financiers font-ils la ville ? Vers un agenda de recherche. EspacesTemps.net 09.07.2013 [en ligne] http://www.espacestemps.net/articles/lesacteurs-des-marches-financiers-font-ils-la-ville/

HALBERT L, ROUANET H (2014) Filtering risk away: global finance capital, transcalar territorial networks and the (un)making of city-regions. Regional Studies 48 (3): 471-484.

Halbert L, Henneberry J, Mouzakis F (2014) Finance, Business Property and Urban and Regional Development. Regional Studies 48 (3): 421-424.

HARVEY D (1989) From managerialism to entrepreneurialism: the transformation of urban governance in late capitalism. Geografiska Annaler 71B: 3-17.

HESSE M (2004) Land for Logistics: Locational Dynamics, Real Estate Markets and Political Regulation of Regional Distribution Complexes. Tijdschrift voor Economische en Sociale Geografie 95 (2): 162-173.

LA LETTRE DE LA PIERRE (2012) Le guide des investisseurs institutionnels. Urbapress/ innovapresse.

Logan J, Moloch H (1987) Urban Fortunes. The Political Economy of Place. University of California Press, Berkeley.

LORRAIN D (2002) Capitalismes urbains : la montée des firmes d'infrastructures. Entreprises et histoire 2002/2 vol. 30 : 7-31.

LORRAIN D (2011a) La main discrète. La finance globale dans la ville. Revue Française de Science Politique 61 (6) : 1097-1122.

LORRAIN D (dir.) (2011b) Métropoles XXL en pays émergents. Presses de Sciences-Po, Paris.

MACLEOD G (2011) Urban politics reconsidered. Growth machine to post-democratic city? Urban Studies 48 (12): 2629-2660.

Masson S, Petiot R (2014) Dynamiques spatiales des activités logistiques d'entreposage et économies d'agglomération : une analyse économétrique. Colloque de l'ASRDLF, MarneLa-Vallée, juillet.

NAPPI-CHOUlET I (2013) La financiarisation du marché immobilier français : de la crise des années 1990 à la crise des subprimes de 2008. Revue d'économie financière 2013/2 (n ${ }^{\circ}$ 110) : 189-206.

NiCHOLLS W J (2005) Power and Governance: Metropolitan Governance in France. Urban Studies, 42 (4): 783-800.

Phelps N A, Parsons N, Ballas D, Dowling A (2006) Business at the margins? Business interests in urban politics. International Journal of Urban and Regional Research 32: $362-383$.

Phelps N A, Wood A (2011) The new postsuburban politics? Urban Studies 48(12): 2591-2610.

PHElPS N A (2012) The Growth Machine Stops? Urban Politics and the Making and Remaking of an Edge City. Urban Affairs Review 48: 670-700.

POLlARD J (2009) Acteurs économiques et régulation politique. Les promoteurs immobiliers au centre des politiques du logement dans les régions de Paris et de Madrid. Thèse de science politique. Sciences-Po Paris, 530 p. 
Raimbault N (2013a) Les firmes de l'immobilier logistique - 1, portrait d'entreprise. Flux $92: 62-74$.

RAimbault N (2013b) Les firmes de l'immobilier logistique - 2. Prologis : "Local partner to global trade". Une firme intégrée et globale dans l'immobilier logistique, portrait d'entreprise. Flux 93/94 : 91-103.

RAIMBAult N (2014) Gouverner le développement logistique de la métropole: périurbanisation, planification et compétition métropolitaine. Le cas du Bassin parisien et éclairages étrangers. Thèse en Aménagement de l'Espace - Urbanisme. Université Paris Est, 520 p. [en ligne] http://www.theses.fr/2014PEST1086/document

Raimbault N, Douet M, Fremont A (2013) Les implantations logistiques entre réseau et territoire. L'Espace Géographique 2013/1 (Tome 42) : 32-43.

RAIMBAULT N, BAHOKEN F (2014) Quelles places pour les activités logistiques dans la métropole parisienne ? Territoire en Mouvement $\mathrm{n}^{\circ} 23-24$ «Villes et industries : crises, recompositions, et nouvelles dynamiques » : 53-74. [en ligne] http://tem.revues.org/2577

Raimbault N, Jacobs W, Van Dongen F (2015) Port regionalization from a relational perspective. The rise of Venlo as Dutch international logistics hub. Tijdschrift voor Economische en Sociale Geografie: sous presse [en ligne] http://onlinelibrary.wiley.com/doi/10.1111/tesg.12134/abstract

Rutland T. (2010) The Financialization of Urban Redevelopment. Geography Compass 4(8): 1167-1178.

Stone C N (1993) Urban Regimes and The Capacity to Govern: A Political Economy Approach. Journal of Urban Affairs 15 (1): 1-28.

SWYNGEDOUW E (2009) The antinomies of the postpolitical city: in search of a democratic politics of environmental production. International Journal of Urban and Regional Studies 33: $601-620$.

TheuriLlat T (2011) La ville négociée : entre financiarisation et durabilité. Géographie, Economie et Société 13 (3) : 225-254.

Theurillat T, Corpataux J, Crevoisier O (2010) Property sector financialization: the case of Swiss pension funds (1992-2005). European Planning Studies 18(2): 189-212.

THEURILlat T, RERAT P, CREVOISIER O (2014) Les marchés immobiliers: acteurs, institutions et territoires. Géographie, économie, société 16(2) : 233-254. 